\title{
Informational Energy and Entropy Applied to Testing Exponentiality
}

\author{
Hadi Alizadeh Noughabi ${ }^{1}$, Havva Alizadeh Noughabi ${ }^{2}$, Jalil Jarrahiferiz ${ }^{3, *}$ \\ ${ }^{1}$ Department of Statistics, University of Birjand, Birjand, Iran \\ 2 Department of Computer Engineering, University of Gonabad, Gonabad, Iran \\ ${ }^{3}$ Department of Mathematics, Birjand Branch, Islamic Azad University, Birjand, Iran
}

\begin{abstract}
The exponential distribution is widely used in reliability and life testing analysis. In this paper, two tests of fit for the exponential distribution based on Informational Energy and entropy are constructed. Consistency and other properties of the tests are proved. Using a simulation study, critical values of the proposed tests are obtained and then power values of tests are computed and compared with each other against various alternatives. Finally, we apply the tests for time between failures of secondary reactor pumps and waiting times for fatal plane accidents in the USA from 1983 to 1998.
\end{abstract}

Keywords Informational energy, Entropy, Monte Carlo simulation, Testing exponentiality, Test power.

AMS 2010 subject classifications 62G10, 62B10

DOI: $10.19139 /$ soic-2310-5070-616

\section{Introduction}

Suppose that the random variable $X$ has distribution function $F$ with density function $f$. The informational energy $\varepsilon(f)$ of the random variable is defined as

$$
\varepsilon(f)=\int_{-\infty}^{\infty} f(x)^{2} d x
$$

Onicescu (1966) justified the name informational energy and its connection to Information Theory in the classical mechanics. Rao (1973) obtained distributions describing equilibrium states in statistical mechanics based on the informational energy. The informational energy has been used in many statistical problems, see Theodorescu (1977), Onicescu and Stefanescu (1979), Pardo and Taneja (1991) and references there in.

In non-parametric statistics, an estimator of informational energy is useful for researcher. Pardo (2003) introduced an estimator of informational energy as follows. He noted that $\varepsilon(f)$ can be expressed as

$$
\varepsilon(f)=\int_{0}^{1}\left(\frac{d}{d p} F^{-1}(p)\right)^{-1} d p .
$$

Then he constructed its estimator by replacing the distribution function $F$ by the empirical distribution function $F_{n}$, and using a difference operator instead of the differential operator. The derivative of $F^{-1}(p)$ is then estimated by a function of the order statistics. Assuming that $X_{1}, \ldots, X_{n}$ is a random sample, the proposed estimator by

*Correspondence to: Department of Mathematics, Birjand Branch, Islamic Azad University, Birjand, Iran. E-mails: alizadehhadi@birjand.ac.ir (H.A.Noughabi); h.alizadeh@gonabad.ac.ir (Havva Alizadeh Noughabi); jarrahi@iaubir.ac.ir (J.Jarrahiferiz).

ISSN 2310-5070 (online) ISSN 2311-004X (print)

Copyright (C) 2020 International Academic Press 
Pardo (2003) is as

$$
\varepsilon_{m n}=\frac{1}{n} \sum_{i=1}^{n} \frac{2 m}{n\left(X_{(i+m)}-X_{(i-m)}\right)},
$$

where $m$ is positive integer, $m \leq \frac{n}{2}$, and $X_{(1)} \leq X_{(2)} \leq \cdots \leq X_{(n)}$ are order statistics of the sample and $X_{(i)}=$ $X_{(1)}$ if $i<1, X_{(i)}=X_{(n)}$ if $i>n$. Consistency of $\varepsilon_{m n}$ is also proved by Pardo (2003).

Pardo (2003) showed that among all distributions that possess a density function $f$ and have a support $(0,1)$, the entropy $\varepsilon(f)$ is minimized by the uniform distribution and based on this property he constructed a test of fit for the uniform distribution. Its test statistic is given as

$$
\varepsilon_{m n}=\frac{1}{n} \sum_{i=1}^{n} \frac{2 m}{n\left(X_{(i+m)}-X_{(i-m)}\right)} .
$$

Large values of $\varepsilon_{m n}$ indicate that the sample is from a non-uniform distribution. Next he obtained the percentage points of the test statistic and power of test by simulation.

Recently, Alizadeh Noughabi and Chahkandi (2015) introduced a test for the normal distribution based on the informational energy and showed that their test has higher power than the competitor tests. Alizadeh Noughabi and Jarrahiferiz (2019) proposed a test of fit for the Laplace distribution and investigated the performance of it. They showed that their test has a good power and confidently can use in practice. Also, Balakrishnan et al. (2004), Balakrishnan et al. (2007), Habibi Rad et al. (2011), and Pakyari and Balakrishnan (2012, 2013), Alizadeh and Balakrishnan (2015), Jarrahiferiz and Alizadeh (2017), and Alizadeh (2017) proposed tests for Type II censored data.

The exponential distribution is widely used in reliability applications and to model data with a constant failure rate. It is often used to model the failure time of manufactured items in production. Some other properties of this distribution are presented in Alizadeh et al. (2019) and Zardasht (2019). Therefore, constructing a goodness of test for this distribution will be useful in practice. In this article, we apply the informational energy and introduce a powerful goodness of fit test for the exponential distribution. Then the properties of the test are stated and compared with the existing other tests.

In Section 2, we introduce two tests of fit for exponentality based on informational energy and entropy, respectively. Consistency and other properties of the tests are established. In Section 3, we obtain critical values and then compute power of the tests against a wide variety of alternatives and show that the test based on informational energy has a good performance. Finally, we analyze two real data sets to illustrate the tests.

\section{Test construction}

In this section, we explain two methods for testing exponentiality.

\subsection{Testing exponentiality based on informational energy}

Suppose $X_{1}, \ldots, X_{n}$ are a random sample from a continuous probability distribution $F$ with density $f$ over a non-negative support and with mean $\mu<\infty$. We are interested to test the hypothesis

$$
H_{0}: f(x)=f_{0}(x)=\lambda \exp (-\lambda x), \quad \text { for all } x \in(0, \infty)
$$

against the general alternative

$$
H_{1}: f(x) \neq f_{0}(x), \quad \text { for some } x \in(0, \infty) \text {. }
$$

where $\lambda=\frac{1}{\mu}$ is unspecified.

Without loss of any generality, by the probability integral transformation $U=F_{0}(X)$, we can reduce the above 
problem of goodness-of-fit, to testing the hypothesis of uniformity on the unit interval. Therefore, if $U_{i}=F_{0}\left(X_{i}\right), i=1,2, \ldots, n$ be the transformed sample, the hypothesis becomes

$$
H_{0}: f(u)=1, \quad 0<u<1
$$

against

$$
H_{1}: f(u) \neq 1, \quad 0<u<1 .
$$

Hence, test of exponentiality convert to test of uniformity.

Here, we apply the test introduced by Pardo (2003) for testing uniformity of the transformed sample, i.e. $U_{i}=F_{0}\left(X_{i}\right), i=1,2, \ldots, n$. Consequently, the proposed test statistic can be stated as

$$
T_{m n}=\frac{1}{n} \sum_{i=1}^{n} \frac{2 m}{n\left(U_{(i+m)}-U_{(i-m)}\right)}=\frac{1}{n} \sum_{i=1}^{n} \frac{2 m}{n\left(F_{0}\left(X_{(i+m)}, \hat{\lambda}\right)-F_{0}\left(X_{(i-m)}, \hat{\lambda}\right)\right)},
$$

where $F_{0}$ is the cumulative distribution function of the exponential distribution, i.e. $F_{0}(x, \hat{\lambda})=1-\exp (-\hat{\lambda} x)$, and $\hat{\lambda}=1 / \bar{X}$. Also, $m$ is a positive integer, $m \leq \frac{n}{2}, X_{(1)} \leq X_{(2)} \leq \ldots \leq X_{(n)}$ are order statistics and $X_{(i)}=X_{(1)}$ if $i<1, X_{(i)}=X_{(n)}$ if $i>n$. We can write

$$
T_{m n}=\frac{2 m}{n^{2}} \sum_{i=1}^{n}\left(\exp \left\{-X_{(i-m)} / \bar{X}\right\}-\exp \left\{-X_{(i+m)} / \bar{X}\right\}\right)^{-1} .
$$

It is obvious that the test statistic is invariant with respect to the scale transformations.

Remark 1

When the parameter of the distribution is specified as $\lambda=\lambda_{0}$, the test statistic is

$$
T_{m n}=\frac{2 m}{n^{2}} \sum_{i=1}^{n}\left(\exp \left\{-\lambda_{0} X_{(i-m)}\right\}-\exp \left\{-\lambda_{0} X_{(i+m)}\right\}\right)^{-1} .
$$

Similar to the argument in Pardo (2003), the following theorems are stated and proved.

\section{Theorem 1}

Let $X_{1}, \ldots, X_{n}$ be a random sample, we have

$$
T_{m n} \geq 1
$$

Proof

We know that the geometric mean does not exceed the arithmetic mean, therefore

$$
\begin{aligned}
T_{m n} & =\frac{2 m}{n^{2}} \sum_{i=1}^{n}\left(\exp \left\{-X_{(i-m)} / \bar{X}\right\}-\exp \left\{-X_{(i+m)} / \bar{X}\right\}\right)^{-1} \\
& \geq \prod_{i=1}^{n}\left(\frac{2 m}{n}\left(\exp \left\{-X_{(i-m)} / \bar{X}\right\}-\exp \left\{-X_{(i+m)} / \bar{X}\right\}\right)^{-1}\right)^{1 / n} \\
& =\exp \left\{\frac{1}{n} \sum_{i=1}^{n} \ln \left(\frac{2 m}{n}\left(\exp \left\{-X_{(i-m)} / \bar{X}\right\}-\exp \left\{-X_{(i+m)} / \bar{X}\right\}\right)^{-1}\right)\right\}=\exp \left\{-U_{m n}\right\} .
\end{aligned}
$$

where

$$
U_{m n}=\frac{1}{n} \sum_{i=1}^{n} \ln \left(\frac{2 m}{n}\left(\exp \left\{-X_{(i-m)} / \bar{X}\right\}-\exp \left\{-X_{(i+m)} / \bar{X}\right\}\right)\right) .
$$


In other hand, we have

$$
\begin{aligned}
\exp \left\{U_{m n}\right\} & =\exp \left\{\frac{1}{n} \sum_{i=1}^{n} \ln \left(\frac{n}{2 m}\left(\exp \left\{-X_{(i-m)} / \bar{X}\right\}-\exp \left\{-X_{(i+m)} / \bar{X}\right\}\right)\right)\right\} \\
& =\prod_{i=1}^{n}\left(\frac{n}{2 m}\left(\exp \left\{-X_{(i-m)} / \bar{X}\right\}-\exp \left\{-X_{(i+m)} / \bar{X}\right\}\right)\right)^{1 / n} \\
& \leq \sum_{i=1}^{n} \frac{\left(\exp \left\{-X_{(i-m)} / \bar{X}\right\}-\exp \left\{-X_{(i+m)} / \bar{X}\right\}\right)}{2 m} \\
& \leq\left(\exp \left\{-X_{(1)} / \bar{X}\right\}-\exp \left\{-X_{(n)} / \bar{X}\right\}\right) \leq 1
\end{aligned}
$$

Therefore,

$$
T_{m n} \geq \exp \left\{-U_{m n}\right\} \geq 1
$$

\section{Theorem 2}

Let $X_{1}, \ldots, X_{n}$ be a random sample from the exponential distribution, if $m=o(n)$ and $m \neq 1$, then

$$
T_{m n} \stackrel{\text { Pr. }}{\longrightarrow} 1 \text { as } n \rightarrow \infty, m \rightarrow \infty .
$$

Proof

Since $Y=F_{0}\left(X_{(i+j)}\right)-F_{0}\left(X_{(i)}\right)$ has a beta distribution with parameters $j$ and $n-j+1$ and

$$
E\left(\frac{1}{Y}\right)=\frac{n}{j-1}
$$

we can obtain $E\left(T_{m n}\right)$ as

$$
\begin{aligned}
E\left(T_{m n}\right) & =\frac{2 m}{n^{2}}\left\{\sum_{i=1}^{m} E\left(\frac{1}{F_{0}\left(X_{(i+m)}, \hat{\lambda}\right)-F_{0}\left(X_{(1)}, \hat{\lambda}\right)}\right)+\sum_{i=m+1}^{n-m} E\left(\frac{1}{F_{0}\left(X_{(i+m)}, \hat{\lambda}\right)-F_{0}\left(X_{(i-m)}, \hat{\lambda}\right)}\right)\right. \\
& +\sum_{i=n-m+1}^{n} E\left(\frac{1}{F_{0}\left(X_{(n)}, \hat{\lambda}\right)-F_{0}\left(X_{(i-m)}, \hat{\lambda}\right)}\right) \\
& =\frac{2 m}{n}\left\{\sum_{i=1}^{m} \frac{1}{i+m-2}+\frac{n-2 m}{2 m-1}+\sum_{i=n-m+1}^{n} \frac{1}{n-i+m-1}\right\} \\
& =\frac{2 m}{n}\left\{2 \sum_{i=1}^{m} \frac{1}{2 m-i-1}+\frac{n-2 m}{2 m-1}\right\} .
\end{aligned}
$$

By using

$$
\sum_{i=1}^{m} \frac{1}{(2 m-1)-i}=\psi(2 m-1)-\psi(m-1),
$$

where $\psi$ is the digamma function, we have

$$
E\left(T_{m n}\right)=\frac{2 m}{n}\left\{2 \psi(2 m-1)-2 \psi(m-1)+\frac{n-2 m}{2 m-1}\right\} .
$$

Since for large value of $x$,

$$
\psi(x) \sim \log x-\frac{1}{2 x}
$$

if $n \rightarrow \infty, m \rightarrow \infty, m=o(n)$ and $m \neq 1$, we have

$$
\lim E\left(T_{m n}\right)=\lim \left\{\frac{4 m}{n} \log \frac{2 m-1}{m-1}+\frac{2 m}{n(m-1)}+\frac{2 m}{2 m-1}-\frac{2 m(2 m+1)}{(2 m-1) n}\right\}=1 .
$$

Therefore,

$$
T_{m n} \stackrel{\text { Pr. }}{\longrightarrow} 1 \text {. }
$$




\subsection{Testing exponentiality based on entropy}

The entropy $H(f)$, of a continuous random variable $X$ with a density function $f(x)$ was defined by Shannon (1948) to be

$$
H(f)=-\int_{-\infty}^{\infty} f(x) \log f(x) d x
$$

Many researchers has been considered the problem of estimation of $H(f)$, including Vasicek (1976), van Es (1992), Ebrahimi et al. (1994), Correa (1995), Yousefzadeh and Arghami (2008), Alizadeh Noughabi (2010), and Alizadeh Noughabi and Arghami (2010).

Let $X_{1}, \ldots, X_{n}$ be a random sample of size $n$, and $X_{(1)} \leq X_{(2)} \leq \ldots \leq X_{(n)}$ denotes the order statistics of sample. Vasicek (1976) first time introduced an estimator of entropy as:

$$
H V_{m n}=\frac{1}{n} \sum_{i=1}^{n} \log \left\{\frac{n}{2 m}\left(X_{(i+m)}-X_{(i-m)}\right)\right\},
$$

where the window size $m$ is a positive integer smaller than $n / 2, X_{(i)}=X_{(1)}$ if $i<1, X_{(i)}=X_{(n)}$ if $i>n$. He proved the consistency of $H V_{m n}$ for the population entropy $H(f)$.

Gokhale (1983) proposed a test statistic for the exponential distribution based on entropy. Then Ebrahimi et al. (1992) obtained a test statistic using Kullback-Leibler information for the exponential distribution. Also, Alizadeh Noughabi and Arghami (2013) showed that the tests based on entropy and Kullback-Leibler information are equivalent. We explain exponentiality test based on entropy as follows.

It is known that if $X$ is a nonnegative random variable and its mean $E(X)=\lambda^{-1}$ is given then

$$
H(f) \leq 1-\log (\lambda),
$$

and among all nonnegative random variables the exponential distribution

$$
f_{0}(x)=\left\{\begin{array}{lc}
\lambda \exp (-\lambda x) & x \geq 0 \\
0 & \text { otherwise }
\end{array}\right.
$$

maximizes $H(f)$ to $H\left(f_{0}\right)=1-\log (\lambda)$. A simple transformation leads

$$
\lambda \exp \left(H\left(f_{0}\right)\right)=e .
$$

Therefore, Gokhale (1983) proposed the following test statistic.

$$
T=\hat{\lambda} \exp (\hat{H}(f)) .
$$

By replacing $\hat{\lambda}=1 / \bar{X}$ and $\hat{H}(f)=H V_{m n}=\frac{1}{n} \sum_{i=1}^{n} \log \left\{\frac{n}{2 m}\left(X_{(i+m)}-X_{(i-m)}\right)\right\}$, we have

$$
T V_{m n}=\frac{\exp \left(H V_{m n}\right)}{\bar{X}}=\frac{n^{2}}{2 m}\left(\sum_{i=1}^{n} X_{i}\right)^{-1}\left[\prod_{i=1}^{n}\left(X_{(i+m)}-X_{(i-m)}\right)\right],
$$

where $H V_{m n}$ is Vasicek entropy estimator and $\bar{X}$ is the sample mean. We reject the null hypothesis for small values of $T V_{m n}$.

\section{Simulation study}

For small to moderate sample sizes, the critical values of the test based on informational energy with 30,000 replications and samples of size $n$ are obtained. Table 1 presents the critical values of the $T_{m n}$-statistic various sample sizes at significance level $\alpha=0.05$. Quantiles of $T V_{m n}$ are reported in Gokhale (1983) and we dont present them.

To comparisons of the power values of the considered tests, we select the same three alternatives listed in Ebrahimi et al. (1992) and their choices of parameters: 
Table 1. Critical values of the $T_{m n}$ statistic at significance level $\alpha=0.05$

\begin{tabular}{ccccccccccc}
\hline & \multicolumn{10}{c}{$m$} \\
\cline { 2 - 11 }$n$ & 1 & 2 & 3 & 4 & 5 & 6 & 7 & 8 & 9 & 10 \\
\hline 5 & 15.19 & 4.579 & & & & & & & & \\
6 & 13.41 & 4.032 & 3.242 & & & & & & & \\
7 & 12.32 & 3.710 & 2.891 & & & & & & & \\
8 & 11.23 & 3.510 & 2.673 & 2.561 & & & & & & \\
9 & 10.28 & 3.246 & 2.531 & 2.380 & & & & & & \\
10 & 9.951 & 3.108 & 2.434 & 2.254 & 2.245 & & & & & \\
15 & 7.343 & 2.589 & 2.052 & 1.912 & 1.903 & 1.848 & 1.886 & & & \\
20 & 6.189 & 2.307 & 1.879 & 1.737 & 1.687 & 1.666 & 1.665 & 1.684 & 1.722 & 1.756 \\
25 & 5.407 & 2.138 & 1.771 & 1.643 & 1.588 & 1.563 & 1.559 & 1.563 & 1.577 & 1.601 \\
30 & 4.934 & 2.028 & 1.683 & 1.566 & 1.517 & 1.497 & 1.489 & 1.490 & 1.497 & 1.507 \\
40 & 4.406 & 1.886 & 1.586 & 1.477 & 1.427 & 1.408 & 1.397 & 1.398 & 1.397 & 1.406 \\
50 & 4.001 & 1.808 & 1.518 & 1.423 & 1.377 & 1.352 & 1.343 & 1.340 & 1.338 & 1.342 \\
\hline
\end{tabular}

(a) the Weibull distribution with density function

$$
f(x ; \lambda, \beta)=\beta \lambda^{\beta} x^{\beta-1} \exp \left\{-(\lambda x)^{\beta}\right\}, \quad \beta>0, \quad \lambda>1, \quad x \geq 0 ;
$$

(b) the gamma distribution with density function

$$
f(x ; \lambda, \beta)=\frac{\lambda^{\beta} x^{\beta-1} \exp \{-\lambda x\}}{\Gamma(\beta)}, \quad \beta>0, \quad \lambda>1, \quad x \geq 0 ;
$$

(c) the log-normal distribution with density function

$$
f\left(x ; v, \sigma^{2}\right)=\frac{1}{x \sigma \sqrt{2 \pi}} \exp \left\{-\frac{1}{2 \sigma^{2}}(\ln (x)-v)^{2}\right\}, \quad-\infty<v<\infty, \quad \sigma^{2}>0, \quad x>0 .
$$

We also chose the parameters so that $E(X)=1$, i.e. $\lambda=\Gamma\left(1+\frac{1}{\beta}\right)$ for the Weibull, $\lambda=\beta$ for the gamma and $v=-\sigma^{2} / 2$ for the log-normal family of distributions.

We compute the power values of the informational energy based test with the power values of the entropy based test, for samples of size equal to 10 and 20. Under each alternative, we generated 20,000 samples of size 10 and 20 and then computed the test statistics $\left(T_{m n}, T V_{m n}\right)$. By the frequency of the event the test statistic is in the critical region the power value of the corresponding test was obtained.

Table 2 presents the estimated powers at significance levels $\alpha=0.01$ and $\alpha=0.05$. The power values of the entropy test are based on the window sizes reported in Ebrahimi et al. (1992), which give the maximum power for this test. For the proposed test, the maximum power was typically attained by choosing $m=5$ for $n=10$, and $m=10$ for $n=20$. Generally, we can say that with increasing $n$ the optimal choice of $m$ increases.

From Table 2, it is seen that the tests are differ in power. It indicates a superiority of the procedure based on informational energy to entropy test. It is observed that for small sample sizes the tests achieve the same power and for large sample sizes the informational energy test has the most power. The difference of power values of the tests $T_{m n}$ and $T V_{m n}$ are substantial.

In the following examples, we use the tests for some real datasets. Histograms of the considered data sets are presented in Figure 1.

\section{Example 1}

Suprawhardana and Sangadji (1999) presented 23 measures corresponding to time between failures (in thousands of hours) of secondary reactor pumps. The data set is

2.160, 0.746, 0.402, 0.954, 0.491, 6.560, 4.992, 0.347, 0.150, 0.358, 0.101, 1.359, 3.465, 1.060, 0.614, 1.921, 
Table 2. Monte Carlo power estimates of the tests

\begin{tabular}{|cc|ccc|ccc|ccc|}
\hline \multirow{2}{*}{ Sample size } & Significance level & \multicolumn{10}{|c|}{ Alternative } \\
\cline { 3 - 10 } & $\alpha$ & \multicolumn{2}{|c|}{ Gamma distribution } & \multicolumn{1}{|c|}{ Weibull distribution } & \multicolumn{2}{c|}{ Log-normal distribution } \\
\hline$n$ & 0.01 & 2 & 0.101 & 0.094 & 2 & 0.345 & 0.330 & -0.3 & 0.083 & 0.084 \\
& 0.05 & & 0.315 & 0.331 & & 0.681 & 0.699 & & 0.285 & 0.322 \\
& 0.01 & 3 & 0.284 & 0.273 & 3 & 0.855 & 0.845 & -0.2 & 0.228 & 0.226 \\
& 0.05 & & 0.627 & 0.652 & & 0.981 & 0.983 & & 0.560 & 0.615 \\
& 0.01 & 4 & 0.485 & 0.482 & 4 & 0.986 & 0.986 & -0.1 & 0.690 & 0.713 \\
& 0.05 & & 0.822 & 0.852 & & 1.000 & 000.1 & & 0.938 & 0.967 \\
& & & & & & & & & & \\
20 & 0.01 & 2 & 0.228 & 0.314 & 2 & 0.734 & 0.829 & -0.3 & 0.198 & 0.323 \\
& 0.05 & & 0.502 & 0.634 & & 0.933 & 0.965 & & 0.475 & 0.671 \\
& 0.01 & 3 & 0.658 & 0.773 & 3 & 0.999 & 1.000 & -0.2 & 0.560 & 0.740 \\
& 0.05 & & 0.889 & 0.954 & & 1.000 & 000.1 & & 0.835 & 0.950 \\
& 0.01 & 4 & 0.898 & 0.958 & 4 & 1.000 & 1.000 & -0.1 & 0.985 & 0.998 \\
& 0.05 & & 0.982 & 0.995 & & 1.000 & 000.1 & & 1.000 & 000.1 \\
\hline
\end{tabular}

4.082, 0.199, 0.605, 0.273, 0.070, 0.062, 5.320.

Here, we computed the values of the test statistics and then compared them with the critical values at the significance level 0.05 and finally we concluded that these data follow an exponential distribution.
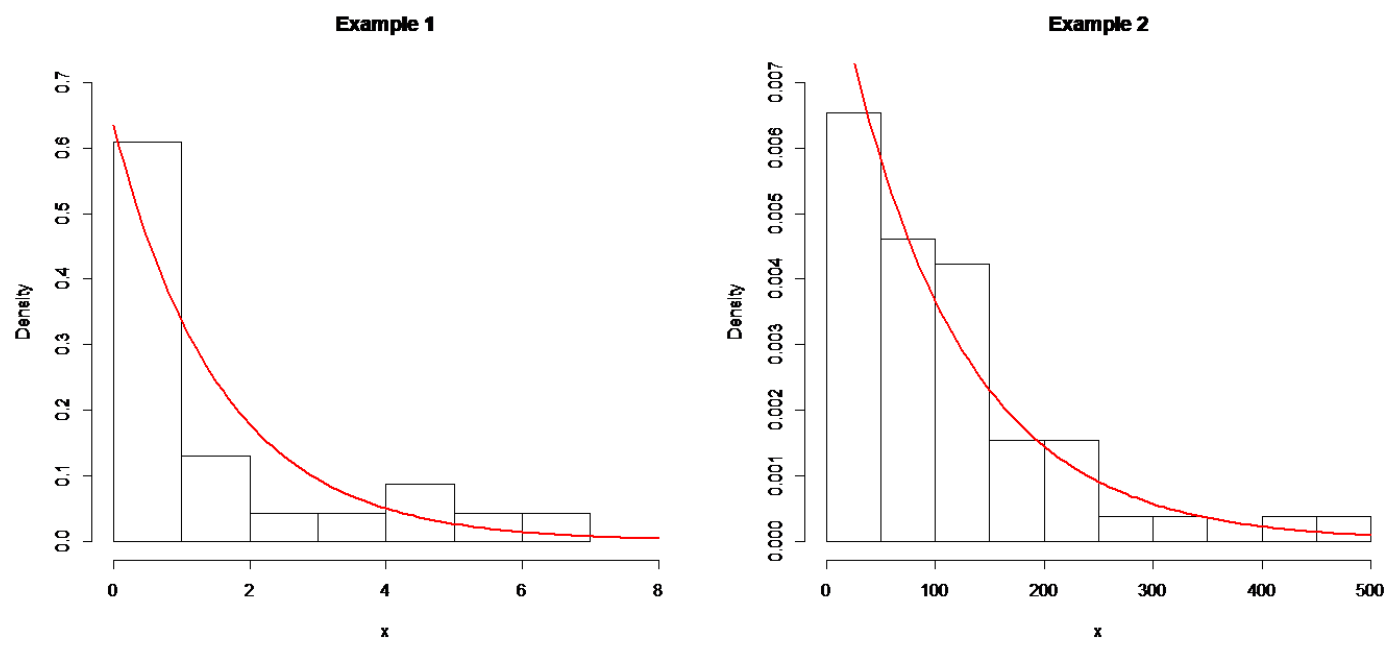

Figure 1. Histograms of the considered data and a fitted exponential density function.

\section{Example 2}

The following data are obtained based on inter-occurrence times in days for fatal accidents suffered by scheduled large planes in the USA from 1983 to 1998. (data from NTSB [14]):

$2,5,7,10,11,13,14,16,17,22,22,22,22,35,36,41,50,53,53,56,60,61,63,63,65,68,70,91,98,112,116$, $117,125,125,127,128,143,143,148,150,151,158,162,194,216,223,236,244,253,310,426,454$.

The proposed tests for goodness of fit on inter-occurrence times of fatal accidents are used. After some computing the values of the proposed tests, we concluded that the distribution of the data of the inter-occurrence times of fatal accidents on scheduled large planes in the USA (1983C1998) does not differ significantly from the exponential. 
Therefore, the inter-occurrence times of fatal accidents suffered by scheduled large planes in the USA from (1983C1998) is exponentially distributed.

\section{Conclusion}

In this paper, we first proposed two tests for exponentaility based on the estimated informational energy and entropy, respectively. Consistency and other properties of the test statistics are presented. Then, we obtained the critical values of the proposed test and also computed the power vales of the considered tests using Monte Carlo computations for different sample sizes against various alternatives. We observed that the test based on informational energy performs very well compared with the test based on entropy for Weibull, gamma, and lognormal alternatives. Also, it can be seen that the relative superiority of the proposed test over entropy test increases with sample size.

\section{REFERENCES}

1. H. Alizadeh Noughabi, A new estimator of entropy and its application in testing normality, Journal of Statistical Computation and Simulation, vol. 80, pp. 1151-1162, 2010.

2. H. Alizadeh Noughabi, Goodness-of-Fit Tests for Lifetime Distributions Based on Type II Censored Data, Journal of Statistical Computation and Simulation, vol. 87, pp. 1787-1798, 2017.

3. H. Alizadeh Noughabi, and N.R. Arghami, General Treatment of Goodness-of-Fit Tests Based on Kullback-Leibler Information, Journal of Statistical Computation and Simulation, vol. 83, pp. 1556-1569, 2013.

4. H. Alizadeh Noughabi, and N. Balakrishnan, Goodness of Fit Using a New Estimate of Kullback-Leibler Information Based on Type II Censored Data, IEEE Transactions on Reliability, vol. 64, pp. 627-635, 2015.

5. H. Alizadeh Noughabi, and M. Chahkandi, Informational energy and its application in testing normality, Annals of Data Science, vol. 2, pp. 391-401, 2015.

6. H. Alizadeh Noughabi, and J. Jarrahiferiz, Informational Energy Based Goodness-of-Fit Test for Laplace Distribution, International Journal of Information and Decision Science, To appear, 2019.

7. M. Alizadeh, M. Emadi, and M. Doostparast, A New Two-Parameter Lifetime Distribution: Properties, Applications and Different Method of Estimations, Statistics, Optimization and Information Computing, vol. 7, pp. 291-310, 2019.

8. N. Balakrishnan, A. Habibi Rad, and N.R. Arghami, Testing exponentiality based on Kullback-Leibler information with progressively type-II censored data, IEEE Transactions on Reliability, vol. 56, pp. 301-307, 2007.

9. N. Balakrishnan, H.K.T. Ng, and N. Kannan, Goodness-of-fit tests based on spacings for progressively Type-II censored data from a general location-scale distribution, IEEE Transactions on Reliability, vol. 53, pp. 349-356, 2004.

10. J.C. Correa, A new estimator of entropy, Communications in Statistics-Theory and Methods, vol. 24, pp. 2439-2449, 1995.

11. N. Ebrahimi, M. Habibullah, and E. Soofi, Testing exponentiality based on Kullback-Leibler information, Journal of the Royal Statistical Society, Series B, vol. 54, pp. 739-748, 1992.

12. N. Ebrahimi, K. Pflughoeft, and E. Soofi, Two measures of sample entropy, Statistics and Probability Letters, vol. 20, pp. 225-234, 1994.

13. D.V. Gokhale, On entropy-based goodness-of-fit test, Computational Statistics \& Data Analysis, vol. 1, pp. 157-165, 1983.

14. A. Habibi Rad, F. Yousefzadeh, and N. Balakrishnan, Goodness-of-fit test based on Kullback-Leibler information for progressively Type-II censored data, IEEE Transactions on Reliability, vol. 60, pp. 570-579, 2011.

15. J. Jarrahiferiz, and H. Alizadeh Noughabi, Testing Exponentiality Using Different Entropy Estimates Based on Type II Censored Data: A Monte Carlo Power Comparison, International Journal of Industrial Engineering, vol. 24, pp. 556-571, 2017.

16. National Transportation Safety Board (NTSB) (scheduled 121...large and sch. 135...small for 1983-1998), http://www.ntsb.gov/ntsb/Response2.asp

17. O. Onicescu, Energie informationelle, C. R. Acad. Sci. Paris. Ser. A, pp. 841-842, 1966.

18. O. Onicescu, and V. Stefanescu, Elemente de Statistica informationala eu aplicatii, Editura Technica, Bucarest, 1979.

19. R. Pakyari, and N. Balakrishnan, A general purpose approximate goodness-of-fit test for progressively Type-II censored data, IEEE Transactions on Reliability, vol. 61, pp. 238-244, 2012.

20. R. Pakyari, and N. Balakrishnan, Goodness-of-fit tests for progressively Type-II censored data from location-scale distributions, Journal of Statistical Computation and Simulation, vol. 83, pp. 167-178, 2013.

21. L. Pardo, and I.J. Taneja, information energy and its applications, Advances in Electronic and Electron Physics (P. Hawkes, Ed.), pp. 165-241, Academic Press, 1991.

22. M.C. Pardo, A test for uniformity based on informational energy, Statistical Papers, vol. 44, pp. 521-534, 2003.

23. C.R. Rao, Linear statistical inference and its applications, John Wiley, New York, 1973.

24. C.E. Shannon, A mathematical theory of communications, Bell System Technical Journal, vol. 27, pp. 379-423; 623-656, 1948.

25. S.M. Suprawhardana and P. Sangadji, Total time on test plot analysis for mechanical components of the RSG-GAS reactor, Atom Indones, vol. 25(2), 1999.

26. A. Theodorescu, Energie informationnelle et notions apparentees, Trabajos de Estadistica e I.O., vol. 27, pp. 276-298, 1977. 
27. B. van Es, Estimating functionals related to a density by class of statistics based on spacings, Scandinavian Journal of Statistics, vol. 19, pp. 61-72, 1992.

28. O. Vasicek, A test for normality based on sample entropy, Journal of the Royal Statistical Society, Series B, vol. 38, pp. 54-59, 1976.

29. F. Yousefzadeh, and N.R. Arghami, Testing exponentiality based on type II censored data and a new cdf estimator, Communications in Statistics-Simulation and Computation, vol. 37, pp. 1479-1499, 2008.

30. V. Zardasht, Results on relative mean residual life and relative cumulative residual entropy, Statistics, Optimization and Information Computing, vol. 7, pp. 150-159, 2019. 